\title{
QCD tests with polarized beams
}

\author{
Takashi Maruy ama ${ }^{a *}$ \\ ${ }^{a}$ Stanford Linear Accelerator Center, Stanford University, Stanford, CA 94309, USA
}

We present three QCD studies performed by the SLD experiment at SLAC, utilizing the highly polarized SLC electron beam. We examined particle production differences in light quark and antiquark hemispheres, and observed more high momentum baryons and $K^{-}$'s than antibaryons and $K^{+}$'s in quark hemispheres, consistent with the "leading particle" hypothesis. We performed a search for jet handedness in light $q^{-}$and $\bar{q}^{-j}$ jets. Assuming Standard Model values of quark polarization in $Z^{0}$ decays, we have set an improved upper limit on the analysing power of the handedness method. We studied the correlation between the $Z^{0}$ spin and the event-plane orientation in polarized $Z^{0}$ decays into three jets. The CP-even and T-odd triple product $\overrightarrow{S_{Z}} \cdot\left(\overrightarrow{k_{1}} \times \overrightarrow{k_{2}}\right)$ formed from the two fastest jet momenta, $\overrightarrow{k_{1}}$ and $\overrightarrow{k_{2}}$, and the $Z^{0}$ polarization vector $\overrightarrow{S_{Z}}$, is sensitive to physics beyond the Standard Model.

\section{INTR ODUCTION}

Since 1992 SLC has been running with a longitudinally polarized electron beam, and SLD has collected approximately 50,000 hadronic $Z^{0}$ decays in 1993 and 100,000 in $1994 / 5$ with average beam polarizations of $63 \%$ and $77 \%$, respectively. In the process $\mathrm{e}^{+} \mathrm{e}^{-} \rightarrow Z^{0} \rightarrow q \bar{q}$, the forward-backward asymmetry $A_{F B}$, quark polarization $P_{q}$, and $\mathrm{Z}^{0}$ polarization $P_{Z^{0}}$ are greatly enhanced by the longitudinal beam polarization as shown in Table 1, and SLD has performed a variety of QCD studies exploiting these features.

Table 1

Expected Asymmetry and Polarization

$$
\begin{array}{ccc} 
& P_{e^{-}}=-77 \% & P_{e^{-}}=+77 \% \\
A_{F B} \text { (u-type) } & +0.42 & -0.35 \\
P_{q} \text { at } \cos \theta= \pm 1 & +0.58 & -0.49 \\
& \mp 0.83 & \pm 0.70
\end{array}
$$

*representing the SLD collaboration

\section{LEADING P ARTICLE PRODUCTION}

In many fragmentation models, the primary quark and antiquark in $Z^{0} \rightarrow q \bar{q}$ events appear in a specific hadron, and this "leading" hadron tends to have large momen tum. Experimentally, by separating quark hemispheres from an tiquark hemispheres, it is possible to look for differences in identified particle production which would be attributable to such "leading" particles. We define a particle to be leading if one of its constituent quarks is a primary quark, for example the $u$ or $\bar{u}$ in $e^{+} e^{-} \rightarrow Z^{0} \rightarrow u \bar{u}$. Then the experimental question is whether the inclusive properties are different for particles that could be "leading" (e.g. a $\pi^{+}$in a $u$-quark jet) and those that could not (e.g. a $\pi^{-}$in a $u$-quark jet). To study this question we must: i) separate $q^{-}$and $\bar{q}$ - jets; ii) select light quark events $\left(Z^{0} \rightarrow u \bar{u}, d \bar{d}, s \bar{s}\right)$ so as to be insensitive to heavy hadron decay products; and iii) identify particle types. We then compare the scaled momen tum distributions of given particles with those of their antiparticle in a pure sample of quark jets in order to determine whether the primary quark prefers a particular particle type or momentum.

The analysis used the charged tracks measured in the central drift chamber [1] and in the vertex detector [2]. A set of cuts was applied to the data to select well-measured tracks, and events were required to be well-contained within the detec- 
tor acceptance. Flavor tagging was performed by counting the number of well measured tracks $n_{\text {sig }}$ that were inconsistent with the interaction point as the origin. Charged particle identification was performed with the Cherenkov Ring Imaging Detector (CRID) [3]. Standard mass reconstruction techniques were used for neutral particle identification [4].

The large forward-backward asymmetry due to the highly polarized electron beam was used to separate quark from antiquark hemispheres. As the primary quarks from $Z^{0}$ decays are left handed, they tend to follow the direction of the incident left-handed fermion. The event was divided in two by a plane perpendicular to the thrust axis, and when the electron beam was left(right)-handed, the hemisphere whose normal satisfied $\vec{n} \cdot \overrightarrow{p_{e}}>0$ was labeled as the quark (antiquark) hemisphere. A cut was applied to remove events whose thrust axis failed to satisfy $\left|\cos \theta_{t}\right|>0.2$, where $\theta_{t}$ is the angle between the thrust axis and the $e^{-}$beam direction. The Standard Model at tree level predicts the purities of the quark- and antiquark-tagged samples to be about $72 \%$ for our average electron beam polarization of $73 \%$.

Light quark events were selected by requiring $n_{\text {sig }}=0$. In each hemisphere, the production rates as a function of $x_{p}$ of identified hadrons were determined. We combined the positively charged tracks in the $q$-tagged jets with the negatively charged tracks in the $\bar{q}$-tagged jets (similarly, $\Lambda$ 's in q-jets were combined with $\bar{\Lambda}$ 's in $\bar{q}-$ jets). We subtracted the contributions to these samples from hea vy quark events, estimated from the Monte Carlo simulation. For each hadron type $h$, the resulting rates were unfolded for the purity of the quark tagging to obtain differential production rates $R(q \rightarrow h)$ in light quark jets.

We define the difference between particle and antiparticle production rates normalized by their sum:

$$
D_{h}=\frac{R(q \rightarrow h)-R(q \rightarrow \bar{h})}{R(q \rightarrow h)+R(q \rightarrow \bar{h})} .
$$

The systematic uncertainties on particle identification largely cancel in this variable, and the errors are dominated by statistics. Figure 1 shows these normalized differences as a function of $x_{p}$.
For each particle type, the differences are consistent with zero at low $x_{p}$. For the $\pi$ 's the difference is also consistent with zero at high $x_{p}$, whereas for the others a significant positive difference is observed for $x_{p}$ above $\sim 0.15$.
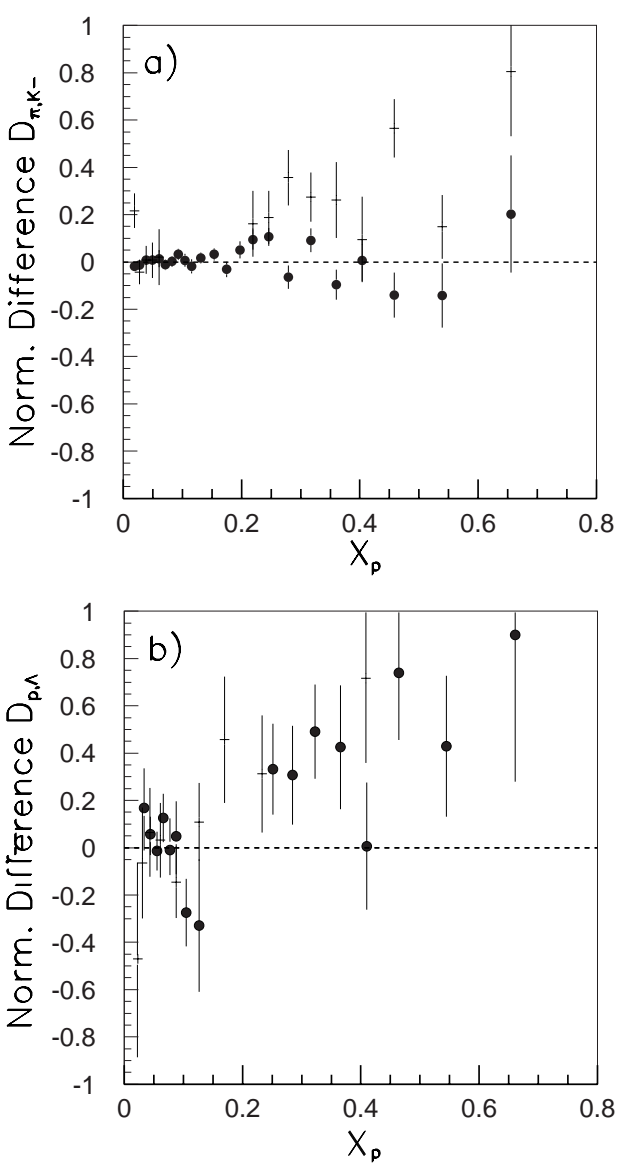

Figure 1. Normalized production differences as a function of scaled momen tum for (a) charged pions (circles) and kaons (crosses), and (b) protons (circles) and $\Lambda$ 's (crosses).

Since the baryons contain no constituent antiquarks, we interpret the steep rise in $D_{p}$ and $D_{\Lambda}$ with increasing $x_{p}$ as an indication that baryon production is dominated by leading particle pro- 
duction as $x_{p} \rightarrow 1$. If production of $\pi^{ \pm}\left(K^{ \pm}\right)$ mesons were dominated by leading meson production, and $\pi^{-}\left(K^{-}\right)$were produced equally in jets containing primary $\bar{u}$ and $d(s)$ quarks, then we would expect to observe normalized differences of $\sim 0.22$ for the mesons, due to (i) the 22:17 production ratio for $Z^{0} \rightarrow d \bar{d}(s \bar{s}): Z^{0} \rightarrow u \bar{u}$, and (ii) the different electroweak asymmetries for $u$ and $d$-type quark production. Our data are more consistent with $D_{\pi}=0$ than $D_{\pi}=0.22$ over the entire measured $x_{p}$ range, suggesting some dilution of leading pions from resonance decays such as the $\rho^{0}$. Our measured $D_{K}$ values above $x_{p} \simeq 0.2$ are consistently above 0.22 , indicating both that $i$ ) there is leading kaon production at high momentum, and $i i$ ) leading kaons are produced more often in $s \bar{s}$ events than in $u \bar{u}$ events.

\section{JET HANDEDNESS}

Polarized incident electrons provide an excellent opportunity to test the transport of spin through the hadronization process since the Standard Model predicts high polarization of quarks from $Z^{0}$ decays with a strong dependence on polar angle and incident electron polarization. A first measurement of spin-transport, using the technique suggested by Ref. 5, was reported in Ref. 6.

This measurement has been updated with the 1994-95 SLD dataset. The simplest observable with the same transformation properties under parity inversion as spin has the form $\Omega=\hat{t} \cdot\left(\overrightarrow{p_{1}} \times\right.$ $\overrightarrow{p_{2}}$ ), where $\hat{t}$ is a unit vector along the jet axis, corresponding to the spin direction of a longitudinally polarized parton, and $\overrightarrow{p_{1}}$ and $\overrightarrow{p_{2}}$ are the momenta of two particles in the jet chosen by some charge-independent prescription, such as $\left|\overrightarrow{p_{1}}\right|>$ $\left|\overrightarrow{p_{2}}\right| .[5]$ A jet may be defined as left- or righthanded if $\Omega$ is negative or positive, respectively. The jet handedness $H$ is then defined as the asymmetry in the number of left- and right-handed jets: $H \equiv\left(N_{\Omega<0}-N_{\Omega>0}\right) /\left(N_{\Omega<0}+N_{\Omega>0}\right)$. Using the expected parton polarization $P_{q}$ from the Standard Model, the analyzing power $\alpha$ of the method is defined by $H=\alpha P_{q}$. The observed $H$ in light-quark jets was found to be consistent with zero, and the $95 \%$ C. L. upper limit of the ana- lyzing power was determined to be $|\alpha|<0.033$. This improves the limit in Ref. 6 by a factor of 3 , and reaffirms that inclusive transportation of spin through the hadronization process is small.

\section{TRIPLE-PR ODUCT CORRELA TION}

For polarized $Z^{0}$ decays to three hadronic jets one can define the triple product $\overrightarrow{S_{Z}} \cdot\left(\overrightarrow{k_{1}} \times \overrightarrow{k_{2}}\right)$, which correlates the $Z^{0}$ boson polarization vector $\overrightarrow{S_{Z}}$ with the normal to the three-jet plane defined by $\overrightarrow{k_{1}}$ and $\overrightarrow{k_{2}}$, the momenta of the highest- and the second-highest energy jets, respectively. The triple product $\overrightarrow{S_{Z}} \cdot\left(\overrightarrow{k_{1}} \times \overrightarrow{k_{2}}\right)$ is even under $\mathrm{C}$ and $\mathrm{P}$ reversals, and odd under $\mathrm{T}_{N}$, where $\mathrm{T}_{N}$ reverses momenta and spin vectors without exchanging initial and final states. Since $\mathrm{T}_{N}$ is not a true time-reversal operation, a non-zero value does not signal CPT violation and is possible in a theory that respects CPT invariance [7]. Similar observables were first proposed for direct experimental observation of the non-Abelian character of QCD in $e^{+} e^{-} \rightarrow \Upsilon \rightarrow g g g$ [8], and in $e^{+} e^{-} \rightarrow q \bar{q} g$ [9] where a sizable signal is expected at c.m. energies $\sqrt{s}$ below $40 \mathrm{GeV}$; no experimental measuremen ts have been performed since a longitudinally polarized electron beam is required.

The differential cross section for $e^{+} e^{-} \rightarrow q \bar{q} g$ for massless quarks may be written $[9,10]$

$\frac{1}{\sigma} \frac{d \sigma}{d \cos \omega}=\frac{9}{16}\left[\left(1-\frac{1}{3} \cos ^{2} \omega\right)+\beta A_{Z} \cos \omega\right]$,

where $\omega$ is the polar angle of the vector normal to the event plane with respect to the electron-beam direction, and $A_{Z}$ is the polarization of the $Z^{0}$ bozon along this same direction $\left(A_{Z}=\left(P_{e^{-}}-\right.\right.$ $\left.A_{e}\right) /\left(1-P_{e^{-}} \cdot A_{e}\right)$, where $P_{e^{-}}$is the electronbeam polarization, defined to be negative for a left-handed beam, and $A_{e}=2 v_{e} a_{e} /\left(v_{e}^{2}+a_{e}^{2}\right)$ with $v_{e}$ and $a_{e}$ the electroweak vector and axial vector coupling parameters of the electron, respectively). With $\left|\beta A_{Z}\right|$ representing the magnitude [11], the second term is proportional to the $\mathrm{T}_{N^{-}}$ odd triple product, and appears as a forwardbackward asymmetry of the event-plane normal relative to the $Z^{0}$ polarization axis. The sign and magnitude of this term are different for the two beam helicities. 
Recently Brandenburg, Dixon, and Shadmi have investigated Standard Model $\mathrm{T}_{N^{-}}$odd contributions of the form $\overrightarrow{S_{Z}} \cdot\left(\overrightarrow{k_{1}} \times \overrightarrow{k_{2}}\right)$ at the $Z^{0}$ resonance [10]. The triple product vanishes identically at tree level [7], but non-zero contributions arise from higher-order processes such as those shown in Fig. 2: (a) QCD rescattering of massive quarks [9], (b) QCD triangle of massive quarks [12], and (c) electroweak rescattering via $W$ and $Z$ exchange loops. Due to various cancellations these contributions are found to be very small at the $Z^{0}$ resonance and yield values of the correlation parameter $|\beta| \lesssim 10^{-5}$. Because of this background-free situation, measurement of the cross section (1) is sensitive to physics processes beyond the Standard Model that give $\beta \neq$ 0 .
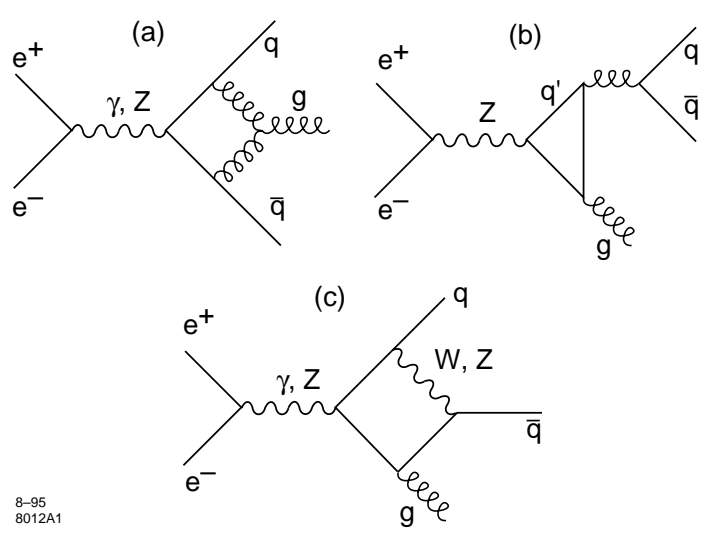

Figure 2. Representative Feynman diagrams of higher-order interactions with non-vanishing contributions to the triple product: (a) QCD rescattering ( $m_{q} \neq 0$ is required), (b) triangle diagram via quark annihilation ( $m_{q}^{\prime} \neq 0$ is required), and (c) electroweak rescattering.

In the analysis [13] the hadronic event selection and three-jet reconstruction were based on the topology of energy depositions in the liquid argon calorimeter, taking advantage of its large solid-angle coverage. The efficiency for selecting hadronic events was estimated to be $92 \pm 2 \%$, with a background in the selected sample of $0.4 \pm 0.2 \%$, dominated by $Z^{0} \rightarrow \tau^{+} \tau^{-}$and $Z^{0} \rightarrow \mathrm{e}^{+} \mathrm{e}^{-}$events.

To measure the triple-product correlation for $e^{+} e^{-} \rightarrow q \bar{q} g$, three-jet events were selected and the three momentum vectors of the jets were reconstructed using the "Durham" jet algorithm [14]. Planar three-jet events were selected by requiring exactly three reconstructed jets to be found with a jet-resolution parameter value of $y_{c}=0.005$, the sum of the angles bet ween the three jets to be greater than $358^{\circ}$, and that each jet contain at least two clusters. A total of 44,683 events satisfied these criteria. The jet energies were calculated by using the measured jet directions and solving the three-body kinematics assuming massless jets, and were then used to label the jets such that $E_{1}>E_{2}>E_{3}$.

For each event the reconstructed jet vectors were used to determine the vector normal to the jet plane and its polar angle $\omega$, from which the measured distribution of $\cos \omega$ was derived. A bin-by-bin correction factor $\epsilon(|\cos \omega|)$, for detector acceptance and initial-state radiation, was determined from Mon te Carlo simulations.

The cosw distribution is described by

$$
\begin{aligned}
\frac{1}{\sigma} \frac{d \sigma}{d \cos \omega} & =\frac{9}{16}\left[\left(1-\frac{1}{3} \cos ^{2} \omega\right)\right. \\
& \left.+\beta A_{Z}\left(1-2 P_{\text {mis }}(|\cos \omega|)\right) \cos \omega\right]
\end{aligned}
$$

where $P_{m i s}$ is the probability of incorrectly ordering the energy of the three jets such that the sign of the $\cos \omega$ term is incorrect. Fig. 3 shows the corrected $\cos \omega$ distribution separately for leftand right-handed beam events in the 1994/5 data sample. A $\mathrm{T}_{N \text {-odd contribution would appear as }}$ a forward-backward asymmetry, of opposite sign between the left- and right-handed events. No asymmetry is apparent. A maximum-likellinood fit of Eq. 2 to the $\cos \omega$ distributions from the 1993 and $1994 / 5$ samples yielded $\beta=0.008 \pm$ 0.015 . The $\mathrm{T}_{N^{-} \text {odd contribution is consistent }}$ with zero within the statistical error and the $95 \%$ C.L. limits are calculated to be $-0.022<\beta<$ 0.039 .

A number of systematic checks were performed. The analysis was performed on two Monte Carlo samples with simulated $\mathrm{T}_{N^{-}}$odd values; in both 


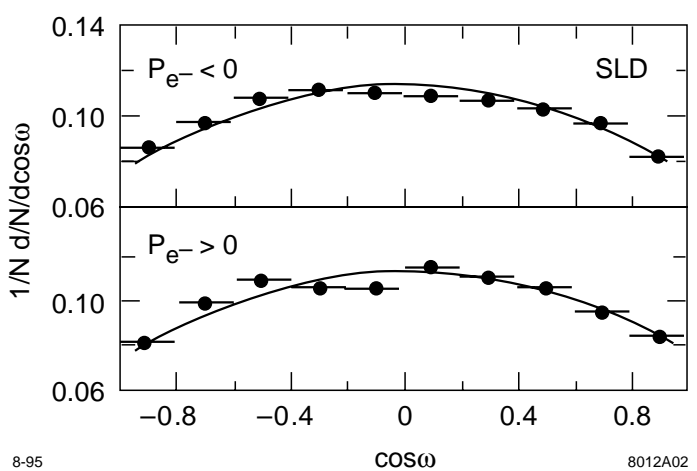

Figure 3. Polar-angle distribution of the jet-plane normal with respect to the electron-beam direction with (a) left-handed and (b) right-handed electron beam. The solid curve is the best fit to the combined data samples.

cases $\beta$ was measured to be consistent with the input value within the statistical error. The dependence of the $\beta$ value on the jet-finding algorithm and the jet-resolution parameter $w$ as examined. The analysis was also performed using only charged tracks measured in the central drift chamber. In each case the $\mathrm{T}_{N^{-} \text {odd }}$ contribution was found to be consistent with zero within the statistical error.

\section{Summary}

We have shown three measurements that exploit the SLC beam polarization and the SLD capabilities. We have measured production rates of $\pi^{ \pm}, K^{ \pm}, p / \bar{p}, K^{0}$ and $\Lambda^{0} / \bar{\Lambda}^{0}$ in light quarkand anti-quark jets, and observed leading baryon and kaon production. We have searched inclusively for spin transport through hadronization, and set limits on jet handedness. Finally, we have measured the $\mathrm{T}_{N^{-}}$odd correlation in polarized $Z^{0}$ decays to three-jets. We find the correlation to be consistent with zero and set $95 \%$ C.L. limits on beyond-the-Standard-Model $\mathrm{T}_{N^{-}}$ odd contributions to $Z^{0}$ decays to three-jets of $-0.022<\beta<0.039$.

This work was supported by Department of Energy Contract No. DE-AC03-76SF00515.

\section{REFERENCES}

1. M.D. Hildreth et al., Nucl. Inst. Meth. A367 (1995) 111.

2. C. J. S. Damerell et al., Nucl. Inst. Meth. A288 (1990) 288.

3. T. J. Pavel, Ph.D. dissertation, Stanford University, in preparation.

4. K.G. Baird, Ph.D. Thesis, Rutgers University, 1996, SLAC-R-483 (1996).

5. O. Nachtmann, Nucl. Phys. B127 (1977) 314 A. V. Efremov et al., Phys. Lett. B284 (1992) 394.

6. K. Abe et al., Phys. Rev. Lett. 74 (1995) 1512.

7. A. De Rújula et al., Nucl. Phys. B35, 365 (1971).

8. A. De Rújula et al., Nucl. Phys. B146, 50 (1978).

9. K. Fabricius et al., Phys. Rev. Lett. 45, 867 (1980), and J. G. Körner et al., Phys. Lett. 94B, 207 (1980).

10. A. Brandenburg, L. Dixon, and Y. Shadmi, Phys. Rev. D53, 1264 (1996).

11. The value of $\beta$ depends on the parameter that determines the jet $m$ ultiplicity of events, and is not a universal constant.

12. K. Hagiwara et al., Nucl. Phys. B358, 80 (1991).

13. K. Abe et al., Phys. Rev. Lett. 75, 4173 (1996).

14. S. Catani et al., Phys. Lett. B263, 491 (1991). 